\title{
Nouveaux procédés utilisés pour le recyclage des eaux dans les charbonnages
}

\author{
PAR \\ E. Condolios \\ Ingénieur Chef du Groupe Ts's \\ ET \\ P. Couratin \\ Ingénieur \\ a Alsthom Technique des Fluides
}

\section{Introduction}

Le charbon esí traité très fréquemment dans les lavoirs par voie humide. En fin de circuit, on retrouve de ce fait des eaux chargées, dites « eaux schlammeuses», dont les schlamms (charbons bruts plus ou moins cendreux) ont des granulométries $0-1 \mathrm{~mm}$, voire davantage jusqu'à $0-10 \mathrm{~mm}$. Le traitement de ces eaux schlammeuses pose des problèmes difficiles surtout si on veut supprimer toute pollution de cours d'eau ou de nappe.

En utilisant de nouveaux procédés, le lavoir de Meyreuil des Houillères de Provence vient d'être équipé de façon à supprimer tous les rejets d'eaux vers la rivière et de bouc au terril ou champ d'épandage. Le présent exposé se pro. pose de décrire les appareils choisis (à l'exception des cyclones qui font l'objet d'une autre communication de M. Condolios). Dans une deuxième partie, la description de l'installation complète est faite.

\section{1 - La clarification des liquides chargés par le procédé Seclar TPM}

\section{1 - PRINCIPE DE FONCTIONNEMENT}

La figure 1 montre le schéma de principe d'un clarificateur du type Seclar TPM.

Cet appareil comprend plusieurs étages ayant chacun une fonction particulière:

-- un foculateur;

- un clarificateur à lit fluidisé;

- un concentrateur.

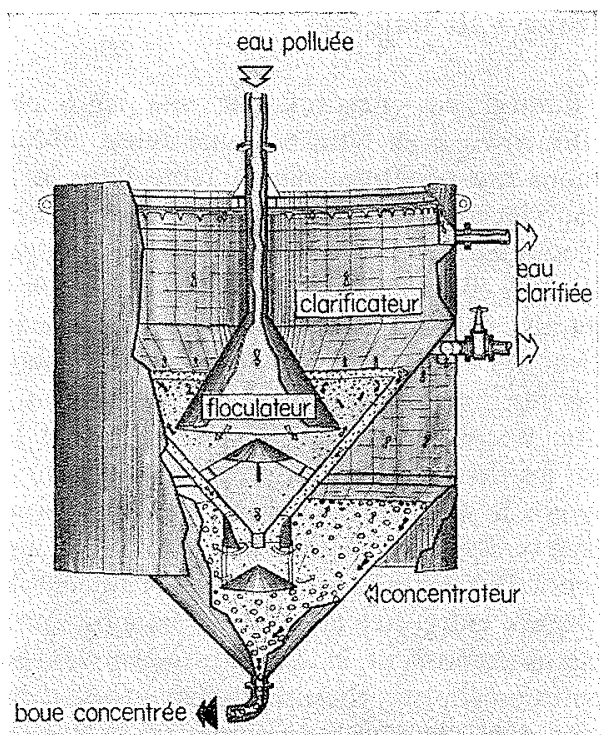

$1 /$

\section{Floculateur}

Le liquide à clarifier arrive par une conduite dans le floculateur primaire.

Avant son introduction dans ce floculateur, une certaine dose d'agents floculants a été mélangée au liquide chargé, de façon à provoquer les rencontres des particules avec les floculants. 


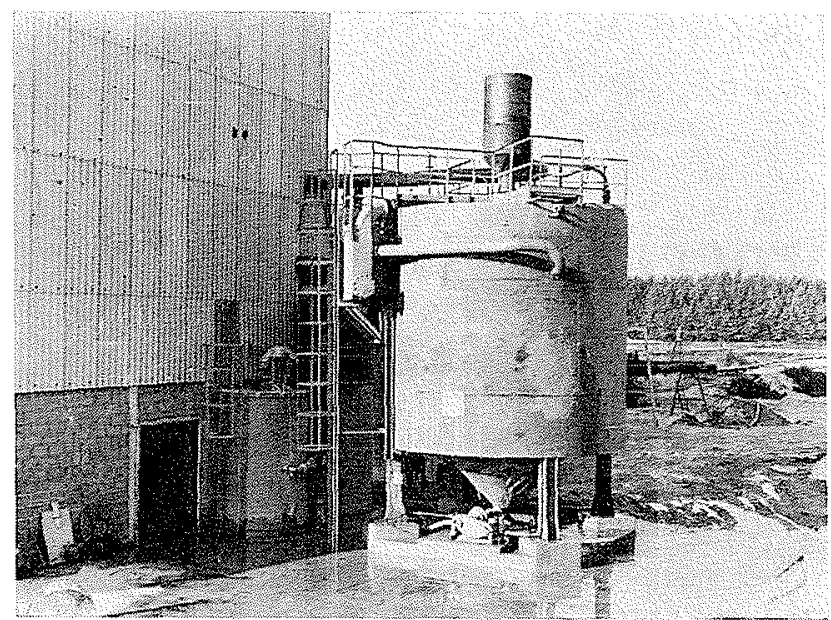

2/

La floculation primaire, déclenchée dans la conduite, se termine dans le floculateur. Dans ce même floculateur, on ajoute alors une nouvelle dose du même agent ou d'un autre agent floculant pour déclencher la floculation secondaire qui va se terminer dans le lit fluidisé du clarificateur.

\section{Clarificateur à lit fluidisé}

Le lit fluidisé est constitué par une zone prismatique conique alimentée à sa base par une fente circulaire.

Les flocons qui pénètrent par la fente dans le lit fluidisé sont encore légers et de faibles dimensions. Ils rencontrent alors un écran de flocons lourds et beaucoup plus gros qui ont grossi dans le lit fluidisé, grâce à un temps de séjour suffisant et aux courants secondaires qu'ils provoquent dans le lit, et qui les ramènent vers le bas. Ces flocons lourds forment un filtre mobile qui piège au passage les particules isolées et les flocons légers qui pénètrent dans le lit fluidisé.

En effet, les fluctuations modérées de l'écoulement turbulent à travers le lit permettent aux flocons d'approcher à une distance convenable les uns des autres. Elles provoquent ainsi, grâce à l'effet conjugué des agents floculants, l'union des flocs légers entre eux et avec les flocons lourds.

Les flocons remontent à des vitesses moyennes de plus en plus lentes au fur et à mesure de leur grossissement, pour ressortir par débordement au sommet de la partie conique.

Le liquide intersticiel qui sort des flocons en haut du lit fluidisé est alors pratiquement clarifié.

Ainsi, grâce à la formation et à l'évolution des plus petits flocons en granulométrie et en densité, on a pu augmenter de façon très importante la vitesse de sédimentation des flocons, donc la vitesse de traversée du courant ascendant à travers le lit fluidisé.

Dans certains cas industriels, cette vitesse près de l'interface, entre le lit de boue et le liquide clarifié, a atteint et même dépassé $100 \mathrm{~m} / \mathrm{h}$.

Le liquide clarifié, sortant du lit de boue fluidisé par la partie cylindrique du clarificateur, peut se déverser en haut de l'appareil par un déversoir à créneaux. Il est évacué par un canal circulaire.

Les flocons lourds qui sortent par débordement du lit fluidisé sont recueillis par un couloir placé à l'extérieur du cône limitant le lit de boue. Ils descendent vers le concentrateur placé à l'étage inférieur.

\section{Concentrateur}

Les flocons lourds, entraînés avec le liquide clarifié, viennent se déposer dans le fond du concentrateur. Ils se tassent avant d'être évacués par l'orifice placé à cet effet en bas de l'appareil.

Le liquide clarifié, libéré par la décantation des flocons, est évacué en haut du concentrateur par un dispositif en couronne équipé de buses adaptées pour réaliser une équirépartition des vitesses de l'écoulement.

La boue concentrée est extraite, en bas du cône du concentrateur, à un débit préréglé de façon à maintenir le lit de boue à une hauteur prédéterminée.

\section{2 - INSTALLATION TYPE DE SECLAR TPM ET PRÉPARATION DU FLOCULANT}

L'installation type telle que le montre la figure 2 se compose :

- du clarificateur Seclar TPM de forme cylindro-conique posé au sol sur des pieds supports;

- des bacs de préparation et de stockage du ou des floculants;

- des pompes de dosage et d'injection des floculants;

- du groupe de pompage pour la reprise des boues du concentrateur.

Une vanne, placée à la sortie du liquide clarifié du concentrateur, permet de régler les débits de liquide clarifié sortant tant du clarificateur que du concentrateur. Des appareils de mesure peuvent être placés dans certains cas pour permettre le contrôle de ces débits.

Les bacs de préparation de floculant sont prévus pour assurer la marche de l'installation pendant un poste de travail de 8 à 10 heures par jour, mais peuvent être conçus et dimensionnés pour un travail continu de trois postes par jour. Les bacs sont équipés de dispositifs de mixage assurant une bonne dispersion et un bon mixage des floculants.

La ou les pompes doseuses à vitesse variable permettent d'injecter, exactement aux points déterminés de la floculation primaire ou secondaire, la dose du ou des floculants les mieux adaptés.

Dans certaines installations, le dosage et le type de floculant doivent être modifiés et ajustés dans le temps en fonction de la provenance différente des matériaux à traiter et de la température.

\section{2 - Le compactage des boues par le procédé Tasster}

La concentration des boues primaires, obtenue après la clarification d'un liquide chargé, est souvent insuffisante et pose presque toujours des difficultés aux utilisateurs quant à leur stockage, leur évacuation ou leur utilisation ultérieure.

En effet, ces boues obtenues par concentration primaire sont généralement peu concentrées de 10 à $30 \%$ de solides 
en poids/poids. Il est souvent indispensable d'augmenter cetle concentration pour diminuer le volume de liquide perdu dans les boues, ou le volume nécessaire à leur stockage ou pour les réintroduire dans un cycle de fabrication.

Grâce à la floculation étagée réalisée dans le Seclar, et à la connaissance des caractéristiques plastiques de ces boues qui, après une nouvelle floculation, sont susceptibles de se surconcentrer, il a été permis de mettre au point un nouveau surconcentrateur qui permet une concentration seconciaire des boues et augmente sensiblement la concentration en solides.

\section{Principe de fonctionnement}

Le surconcentrateur Tasster (fig. 3) est essentiellement constitué d'un bac cylindrique avec un fond conique, dans lequel la boue à surconcentrer est injectée dans la partie centrale avec une certaine quantité de floculant.

Les flocons formant la boue arrivent près d'un jeu de pales de formes et d'inclinaisons appropriées portant des ouvertures; ces pales poussent les flocons à une vitesse déterminée, inférieure à la vitesse de cisaillement.

Les flocons ainsi agités mécaniquement se rapprochent les uns des autres, le liquide intersticiel entre flocons passe à travers les orifices des pales et remonte vers la surface.

Deux jeux de pales et de contre-pales, placés judicieusement dans l'appareil, permettent de parfaire le compactage des flocons avant leur introduction dans une vis conique tournant dans la partie conique inférieure de l'appareil.

Cette vis conique, de pas variable, porte également des orifices appropriés pour, d'une part rapprocher encore les flocons par tassement mécanique et, d'autre part, extraire le liquide et lui permettre de remonter vers la surface.

La boue ainsi compactée peut être extraite, soit par un orifice placé en bas de l'appareil, soit par une pompe volumétrique. Le liquide clarifié extrait des boues surconcentrées surverse par le haut de l'appareil.

\section{3 - Description de l'installation de traitement des eaux schlammeuses du lavoir de Meyreuil}

\section{1 - CARACTÉRISTIQUES DES EAUX A TRAITER}

Les eaux à traiter résultant de l'arrosage de tous les appareils du lavoir sont collectées dans une cuve (cuve 106).

- Débit d'eau à traiter : $500 \mathrm{~m}^{3} / \mathrm{h}$ environ.

- Contenu de ces eaux : 40 à $60 \mathrm{t} / \mathrm{h}$ de charbon brut $0-10 \mathrm{~mm}$ dont 3 à $12 \mathrm{t} / \mathrm{h}$ d'inférieurs à 80 microns.

\section{2 - SCHÉMA DE TRAITEMENT}

Le schéma de traitement (fig. 4) comporte trois étages successifs :

- un cyclonage-essorage pour récupérer les grains $80 \mathrm{mi}$ crons-10 mm;

- un décanteur Seclar permettant de récupérer :

- d'une part de l'eau claire recirculée dans le lavoir,

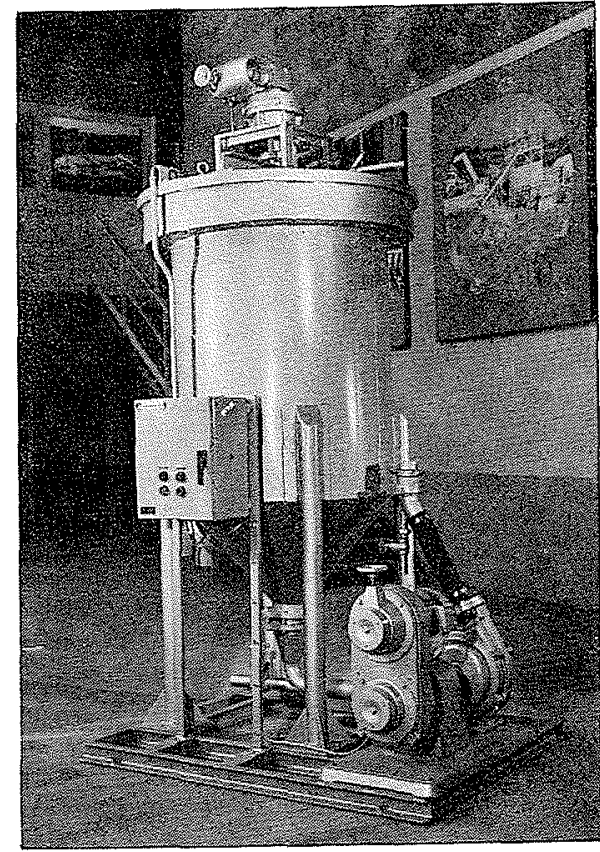

3/ - d'autre part des boues contenant le charbon
0-80 microns;

- un ensemble de Tasster compactant les boues de façon à les envoyer vers la centrale thermique, les eaux récupérées aux Tasster étant aussi recyclées.

\section{3 - PRODUITS SORTANT DE L'INSTALLATION DE TRAITEMENT}

Cette installation permet de ne rejeter aucune eau à l'exception de celle perdue par l'humidité des charbons extraits des eaux schlammeuses et celle des charbons lavés.

Les deux sorties de l'installation délivrent:

- 40 à $50 \mathrm{t} / \mathrm{h}$ de charbon brut 80 microns- $10 \mathrm{~mm}$ à un taux d'humidité de $22 \%$;

- 3 à $12 \mathrm{t} / \mathrm{h}$ de charbon fin $0-80$ microns à une humidité de 50 à $53 \%$.

\section{4 - CARACTÉRISTIQUes DES APPAREILS}

\subsection{1 - Cyclonage}

Le circuit de cyclonage fonctionne avec un débit de $650 \mathrm{~m}^{3} / \mathrm{h}$ (dont $150 \mathrm{~m}^{3 / \mathrm{h}}$ recyclés à la cuve 106). Il comporte :

- une pompe centrifuge Ensival, débit $650 \mathrm{~m}^{3} / \mathrm{h}$;

- deux batteries de trois cyclones $\varnothing 350 \mathrm{~mm}$ en fonte Ni-Hard, équipés de basculeurs;

- deux essoreurs Chauvin EV 36 alimentés chacun par une batterie de cyclones:

- surface de grille: $3,6 \mathrm{~m}^{2}$ (par appareil),

- maille : $0,6 \mathrm{~mm}$. 


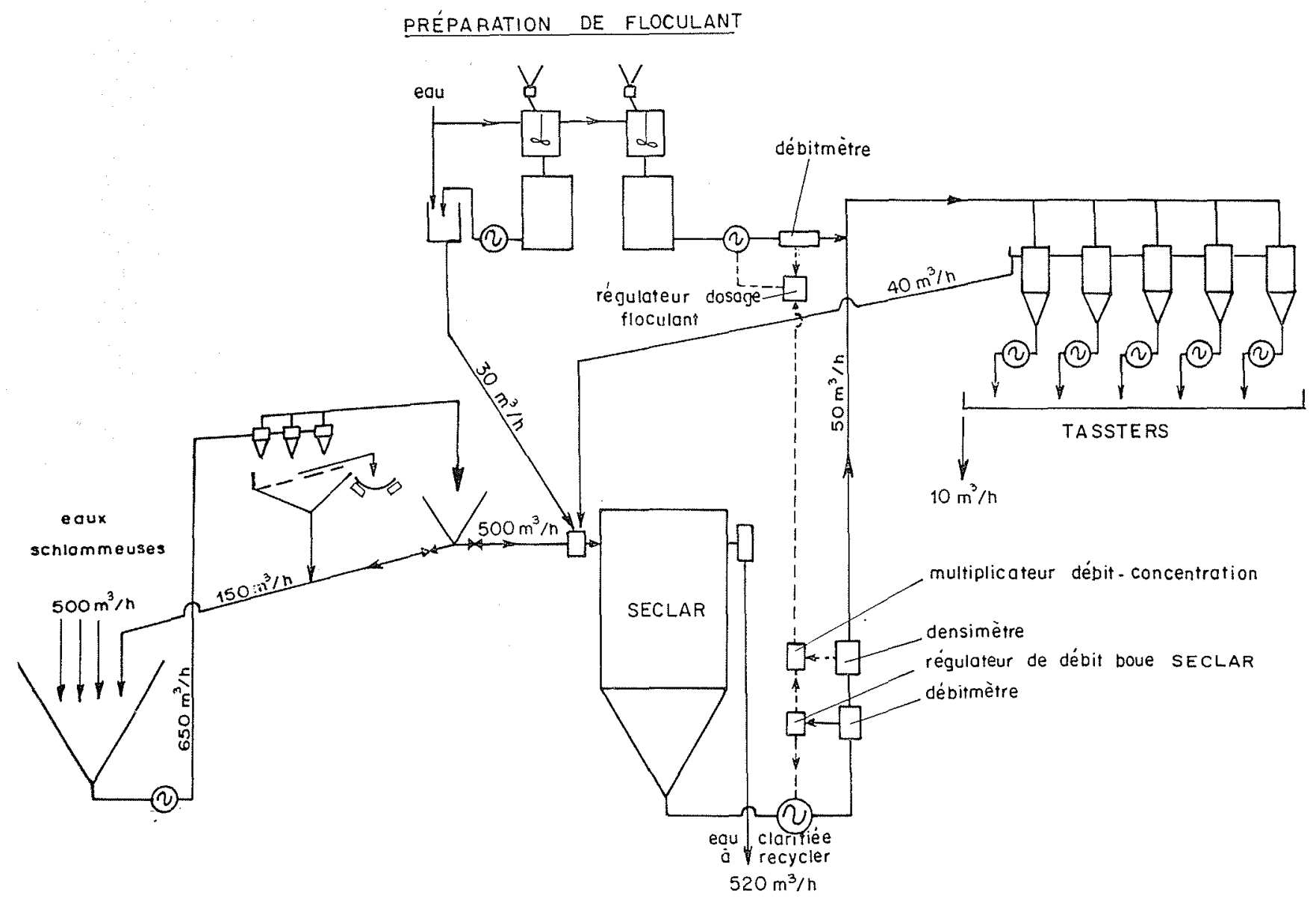

4) Laverie de Mayreuil : Schéma de traitement des eaux schlammeuses.

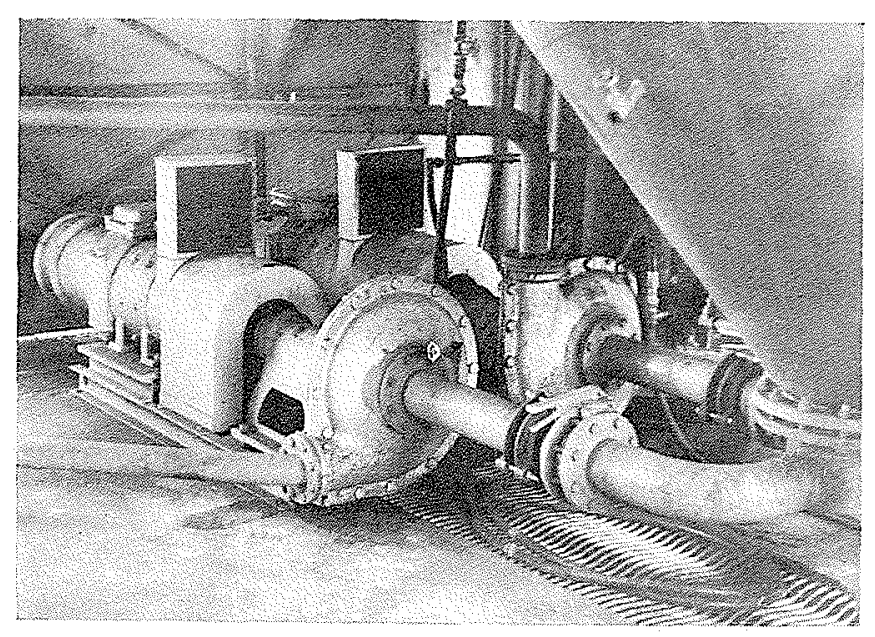

5/

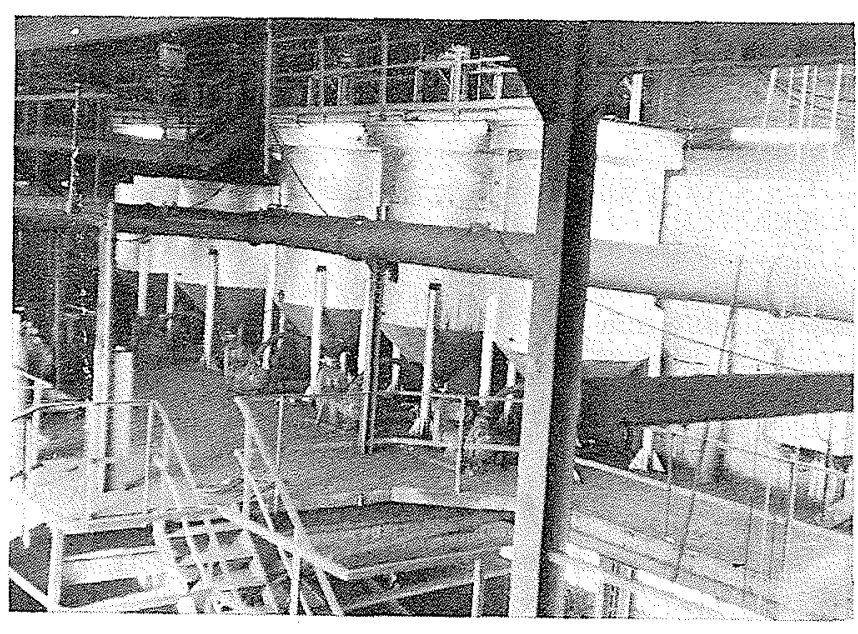

6/ 


\subsection{2 - Décanteur Seclar}

Dimensionné pour le débit maximal prévu de $650 \mathrm{~m}^{3} / \mathrm{h}$, le décanteur Seclar a pour dimensions principales:

- diamètre : $6,5 \mathrm{~m}$;

- hauteur : $9 \mathrm{~m}$;

- vitesse de décantation : $20 \mathrm{~m} / \mathrm{h}$.

Les boues extraites au pied du Seclar ont une concentration ajustable entre 50 et $400 \mathrm{~g} / \mathrm{l}$ suivant le débit d'extraction choisi. Pratiquement, on adopte une concentration comprise entre 100 et $200 \mathrm{~g} / \mathrm{l}$ qui est la concentration la plus favorable à la floculation effectuée à l'entrée des Tasster. Pour cela, le débit d'extraction est ajusté entre 20 et $75 \mathrm{~m}^{3} / \mathrm{h}$ (fig. 5 ).

Le Seclar est alimenté par une station de préparation de floculant automatique à un dosage de 1,5 à $2 \mathrm{ppm}$ ( 1,5 à $2 \mathrm{~g}$ de floculant sec par mètre cube d'eau traitée).

\subsection{3 - Tasster}

Pour la capacité maximale prévue de $15 \mathrm{t} / \mathrm{h}$ de charbon fin, on a choisi quatre Tasster 18 plus un appareil de secours. Les caractéristiques de ces appareils (fig. 6) sont :

- diamètre : $1,8 \mathrm{~m}$;

- hauteur : $2,4 \mathrm{~m}$;

- vitesse de rotation de l'agitateur : $0,3 \mathrm{tr} / \mathrm{mn}$ (entrainée par un moto-réducteur Sadi-Brown avec moteur 0,5 ch).

Chacun des Tasster est équipé d'une pompe d'extraction type Delasco Ly 50 D (fig. 7) dont les caractéristiques sont :

- diamètre de la tubulure : $50 \mathrm{~mm}$;

- puissance du moteur : $3 \mathrm{ch}$;

— vitesse de rotation utilisée : 80 à $100 \mathrm{tr} / \mathrm{mn}$;

- débit instantané de boue épaisse extraite: 5 à $6 \mathrm{~m}^{3} / \mathrm{h}$ (marche intermittente).

La consommation en fioculant des Tasster est d'environ $400 \mathrm{~g} / \mathrm{t}$ de charbon sec.

\subsection{4 - Station de préparation de floculant} (fig. 8)

Le Seclar et l'ensemble des Tasster disposent chacun d'une station de préparation de floculant, ce qui permet le fonctionnement avec deux types de floculants différents dans certains cas, un foculant donnant une bonne clarification dans le Seclar et un floculant de tassement pour les Tasster.

Chacune de ces stations est automatique. Elle comprend:

- un doseur de floculant à vis distribuant $8 \mathrm{~kg}$ de floculant en poudre en $25 \mathrm{mn}$ pour chaque préparation;

- un bac agité de 2,6 $\mathrm{m}^{3}$ équipé d'un agitateur SEM qui reçoit simultanément l'eau claire et le floculant en poudre. Le temps d'une préparation est de $2 \mathrm{~h}$. La préparation est faite à $3 \%$;

- un bac de stockage de $9 \mathrm{~m}^{3}$ qui reçoit le floculant prêt à l'emploi et commande la mise en service automatique du groupe de préparation dès que son niveau permet de recevoir $2,6 \mathrm{~m}^{3}$ de solution.

\section{5 - FONCTIONNEMENT - ASSERVISSEMENT REGULATION}

Le fonctionnement de l'installation ne présente pas de séquences particulières. Dès que le lavoir se met en service, tous les appareils sont alimentés électriquement et se mettent en service, sauf les pompes d'extraction des Tasster qui ne fonctionnent que si le niveau de boue dans l'appareil le justifie. La seule sélection faite est celle des quatre Tasster à exploiter sur les cinq disponibles.

\subsection{1 - Réglage du débit de floculant Seclar}

Le réglage du débit de floculant du Seclar est fait manuellement par action sur le variateur de vitesse de la pompe doseuse Moineau PRF 2 D. Ce réglage n'intervient
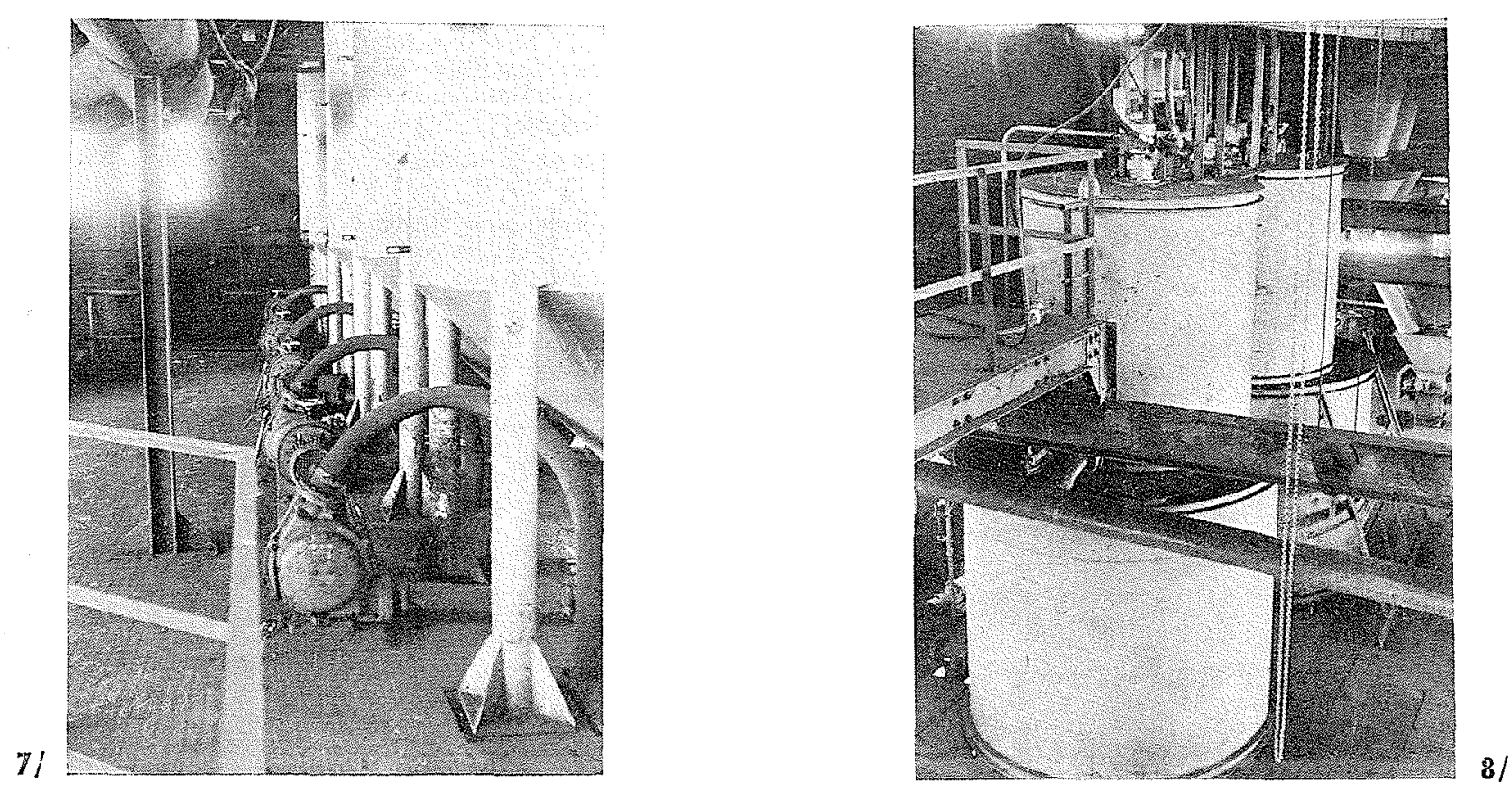
que très rarement, lors des changements importants dans la qualité du charbon.

\subsection{2 - Régulation d'extraction du débit de boue du Seclar}

Le débit d'extraction des boues du Seclar est réglé entre 20 et $75 \mathrm{~m}^{3} / \mathrm{h}$ suivant le tonnage de charbon reçu et le nombre de Tasster en service. La variation est obtenue par le réglage de la vitesse de rotation du moteur à courant continu d'entraînement de la pompe.

Un débitmètre électromagnétique Foxboro mesure la valeur effective du débit qui est comparée, dans un régulateur, avec la valeur de consigne et ajustée en conséquence. Depuis la salle de contrôle du lavoir, il suffit au chef de poste d'afficher le débit désiré pour que celui-ci soit maintenu constant malgré les variations de concentration qui réagissent sur les pertes de charge de la conduite de liaison entre Seclar et Tasster.

\subsection{3 - Régulation du dosage de floculant dans les Tasster}

Le bon fonctionnement des Tasster est obtenu avec un dosage de floculant proportionnel au débit solide de charbon et non au débit de boue introduit. Cette relation de proportionnalité est obtenue par un ensemble de mesures et un dispositif de régulation.

La mesure du débit de boues sortant du Seclar est faite par un débitmètre électromagnétique Foxboro comme rappelé plus haut. La mesure de concentration est exécutée par un gamma-densimètre Schlumberger. Le produit de ces deux mesures, introduit sur un indicateur, fait apparaître le débit solide.

Un régulateur recevant ce même signal affiche le débit de floculant à injecter, commande la vitesse de rotation de la pompe doseuse et compare le débit demandé avec le débit de floculant mesuré par un deuxième débitmètre électromagnétique.

\section{Discussion}

Président: M. P. BergeroN

M. le Président remercie M. Couratin de son intéressant exposé et ouvre la discussion.

Sur une question de M. Misson, M. Couratin indique que les charbons récupérés dans le Tasster sont envoyés tels quels à la centrale électrique où ils sont brûlés avec les autres «fines》 dont la teneur en humidité n'est pas sensiblement modifiée par l'apport d'un faible tonnage de boue.

Quelle est la consommation en poly-b́lectrolytes? demande M. Minart.

Elle est d'environ $400 \mathrm{~g}$ par tonne dans les Tasster et de $200 \mathrm{~g}$ par tonne dans le Seclar; ces réactifs ne présentent, ici, aucun inconvénient puisque l'installation ne rejette aucun effluent, répond M. Couratin.

M. Misson s'enquiert de la possibilité de récupérer - par les procédés décrits - les résidus de charbon contenus dans les terril si nombreux dans les régions houillères. Mais, dit $M$. Couratin. les terrils sont souvent «brûlés» par combustion spontanée des pyrites et des schistes houillers qu'ils contiennent; d'autre part, il n'est pas évident qu'une telle récupération soit rentable.

Quelle est la teneur en humidité des boues à la sortie du Seclar et du Tasster? interroge M. Martin.

A la sortie du Seclar, répond M. Couratin, nous pouvons arriver à $400 \mathrm{~g} / 1$ de matières solides..., mais cela n'est guère nécessaire si les boues sont ensuite traitées au Tasster qui extrait environ $50 \%$ d'humidité.

Sur une question de M. Thevenin, M. Couratin précise que Thumidité est définie suivant la tradition minière par le rapport: poids d'eau/poids de matière humide; cela donne évidemment uil chiftre très différent de celui calculé par rapport an poids de matière sèche.

Le coût de linstallation décrite est de lordre de 2 millions de francs. Le floculant utilisé (à raison de $600 \mathrm{~g}$ par tonne) vaut environ 20 francs le kilo.

En l'absence d'autres interventions, M. le Président clôt la discussion en remerciant tous ceux qui y ont participé. Il donne ensuite la parole à M. Rovel pour l'exposé de sa communication. 